\title{
An Improved Algorithm for Optimal Solution of Unbalanced Transportation Problems
}

\author{
Beena Gill $^{1}$ Dr. M. Anwar Solangi ${ }^{2}$ Dr. A. Sami Qureshi ${ }^{3}$ \\ Department of Basic Sciences and Related Studies, Mehran University of Engineering and Technology, \\ Jamshoro, Sindh, Pakistan
}

\begin{abstract}
Unbalanced transportation problems are particular kind of transportation problems, but an optimal solution is hard to find for unbalanced transportation problems. Still there is a need for minimizing the transportation cost. Unbalanced-TP deals with two different cases, (i) Excess of accessibility $\sum_{\mathbf{i}} \mathbf{A}_{\mathbf{i}}>\sum_{\mathbf{j}} \mathbf{B}_{\mathbf{j}}$, (ii) Deficiency in accessibility $\sum_{\mathbf{i}} \mathbf{A}_{\mathbf{i}}<\sum_{\mathbf{j}} \mathbf{B}_{\mathbf{j}}$, here in this paper both the cases for getting better optimal solution are discussed. Proposed algorithm is based on dummy rows and dummy columns, by taking the absolute differences (penalty) of Initial \& Last cost cells of each row/column in transportation cost-matrix, where the objective function is to find an optimal solution. This method is easy to understand and apply than the other existing methods using Initial Basic Feasible Solution-IBFS. Therefore, the proposed method is very helpful to get optimal solution for unbalanced transportation problems.
\end{abstract}

Keywords:Initial Basic Feasible Solution-IBFS, Unbalanced Transportation Problems, Dummy Rows \& Dummy Columns, Optimal Solution.

DOI: $10.7176 / \mathrm{MTM} / 10-8-02$

Publication date: December $31^{\text {st }} 2020$

\section{INTRODUCTION}

In Operation Research-OR, Transportation Problem-TP is specific kind of sub-division of linear programmingLP problems. It is commonly significant in the scheme of decision making [1]. Wherein purpose is to lessen the cost of transportation for businesses with number of destination, while sustaining supply limit and demand prerequisite [2]. To get this aim of cost, we have the quantity and locality of already accessible supplies and the amount demanded on top of the participation united accompanied by each 'sending'. The term 'Transportation Model' is some time deceive because it can be used for plant location, machine assignment, product mix problems and many more as well. Whereas the model is really not limited to transportation/distribution only [5]. Linear programming is a mathematical technique which is widely used in fields of social sciences and business domain. For example: Transportation Problems, Allocation Problems, Diet Problems, Agriculture Problems, Network Flow Problems, Marketing Engineering and several others. L.F. Hitchcock (1941) initially established basic transportation problems. The motive of transportation problem controls optimal distribution arrangements between origins/sources and destinations, which are basically accumulated at many origins to diverse destinations in such a way that the cost of total transportation is minimized $[4,7,10]$. There are two forms of transportation problems.

\subsection{Balanced and Unbalanced Transportation Problem}

A transportation problem is known as balanced when the summation of all supply bases are equal to the summation of all demand purposes, i.e. $\sum_{\mathrm{i}=\mathbf{1}}^{\mathrm{m}} \mathbf{A}_{\mathbf{i}}=\sum_{\mathrm{j}=\mathbf{1}}^{\mathrm{n}} \mathbf{B}_{\mathrm{j}}$ otherwise it is known as unbalanced transportation problem, i.e. $\sum_{i=1}^{m} \mathbf{A}_{i}>\sum_{j=1}^{n} \mathbf{B}_{j}$ or $\sum_{i=1}^{m} \mathbf{A}_{i}<\sum_{j=1}^{n} \mathbf{B}_{j}$, to satisfy both the conditions of unbalanced transportation problems, they are presented with a dummy row or dummy column. If the sum of sources is greater than the sum of requirements, then the dummy destinations are presented with requirements, to solve the difference between sum of sources and sum of requirements cost, the dummy rows and dummy columns are set equal to zero transportation cost $[6,8]$.

In previous, studies many researchers have discussed different methods to solve the transportation problems of Initial Basic Feasible Solution-IBFS and optimal methods. The most advantageous and useful ways for initial basic feasible solution are North-West Corner Method-NWCM, Least-Cost Method-LCM, Vogel's Approximation Method-VAM and several other. Other methods like Modified Distribution-MODI Method and Stepping Stone Method-SSM are used for an optimal solution of transportation problems. Some important related works in last few years has dealt with. Z.A.M.S. Juman \& N.G.S.A. Nawarathne [8] Considered only row penalties using Juman and Hoque (2015)'s Method-JHM, for solving the I.B.F.S of conservative transportation problem. A. Rashid [9] He proposed a different approach which can help the decision makers in handling unbalanced transportation assignment problems to minimize the costs of total transportation and maximize the total profits. A. Sridhar \& N. Girmay, et al $[11,12]$ suggests a Heuristic Approach to get improved results of 
unbalanced transportation problems, and improved the existing VAM. This study has minimized the transportation costs in an easy and effective manner. A. Quddoos, et al [13] has proposed method named ASMMethod to find optimal solution of transportation problems, directly. This method requires easy arithmetical and logical calculation with less time period, and results are comparable to existing MODI-Method and Stepping Stone Method. Rajendra B. Patel [14] the presented method is a little modification of ASM-Method. M. Palanivel, et al [15] analyzed an innovative procedure for solving problems of transportation by using Harmonic Mean Approach. Ravi Kumar R, et al [16] they proposed a new technique named Direct Sum Method using VAM, to discover the I.B.F.S of transportation problems. A. Seethalakshmy \& N. Srinivasan [17] proposed a different technique to resolve of unbalanced transportation assignment problems, based on finding the position of ' 1 ' by using systematic procedure. This article gives same result as that of the Hungarian Method with less consumption of time. M. A. Metlo, et al [18] Modified North-West Corner Method-MNWCM is developed to discover I.B.F.S based on NWCM. The main objective of this study is to reduce the size of iterations and is used to achieve an optimal solution with minimum time estimation, also the results are compared with existing NWCM.

This paper is a motivation for finding better optimal solution for unbalanced transportation problems. This leads to a different approach than the previous methods to minimize the total cost and to maximize the profit in transporting commodities from sources to destinations. The proposed method is introduced below:

\section{METHODOLOGY:}

\subsection{Mathematical Formulation of Unbalanced Transportation Model:}

Mathematically, the general transportation model is given as follows:

$$
\begin{aligned}
& \text { Minimize: (Total transportation cost) } \mathbf{Z}=\sum_{\mathrm{i}=\mathbf{1}}^{\mathrm{m}} \sum_{\mathbf{j}=\mathbf{1}}^{\mathrm{n}} \mathbf{C}_{\mathrm{ij}} \mathbf{U}_{\mathbf{i j}} \\
& \text { Subject to: (Supply from sources) } \quad \sum_{\mathbf{i}=\mathbf{1}} \mathbf{U}_{\mathbf{i j}} \leq \mathbf{A}_{\mathbf{i}} ; \quad \mathbf{i}=\mathbf{1}, \mathbf{2}, \ldots \ldots, \mathbf{m} \\
& \text { (Demand from destinations) } \sum_{\mathbf{j}=\mathbf{1}}^{\mathrm{n}} \mathbf{U}_{\mathbf{i j}} \geq \mathbf{B}_{\mathbf{j}} ; \quad \mathbf{j}=\mathbf{1}, \mathbf{2}, \ldots \ldots, \mathbf{n} \\
& \text { Where } \quad \mathbf{U}_{\mathbf{i j}} \geq \mathbf{0} ; \quad \forall \mathbf{i} \& \mathbf{j} \\
& \text { (For an unbalanced transportation problem) } \quad \sum_{\mathbf{i}=\mathbf{1}}^{\mathbf{m}} \mathbf{A}_{\mathbf{i}} \neq \sum_{\mathbf{j}=\mathbf{1}}^{\mathbf{n}} \mathbf{B}_{\mathbf{j}}
\end{aligned}
$$

The unbalanced transportation problems are of two cases (i) $\sum_{\mathbf{i}} \mathbf{A}_{\mathbf{i}}>\sum_{\mathbf{j}} \mathbf{B}_{\mathbf{j}}$ and (ii) $\sum_{\mathbf{i}} \mathbf{A}_{\mathbf{i}}<\sum_{\mathbf{j}} \mathbf{B}_{\mathbf{j}}$. There are two feasible cases:

$$
\text { i. } \quad \begin{array}{ccc}
\text { (Excess accessibility) } & \sum_{\mathbf{i}=\mathbf{1}}^{\mathbf{m}} \mathbf{A}_{\mathbf{i}}>\sum_{\mathbf{j}=\mathbf{1}}^{\mathbf{n}} \mathbf{B}_{\mathbf{j}} \\
& (\text { A dummy purpose) } & \mathbf{D}_{\mathbf{n}+\mathbf{1}} \\
\mathbf{U}_{\mathbf{i}, \mathbf{n}+\mathbf{1}}=\mathbf{0} ; \quad \mathbf{i}=\mathbf{1}, \mathbf{2}, \ldots \ldots, \mathbf{m}
\end{array}
$$

Necessary condition for presence of a feasible solution of unbalanced transportation problems are $\sum_{\mathbf{i}=\mathbf{1}}^{\mathbf{m}} \mathbf{A}_{\mathbf{i}}=$ $\sum_{\mathbf{j}=\mathbf{1}}^{\mathbf{n}+\mathbf{1}} \mathbf{B}_{\mathbf{j}}$ or $\sum_{\mathbf{i}=\mathbf{1}}^{\mathbf{m}+\mathbf{1}} \mathbf{A}_{\mathbf{i}}=\sum_{\mathbf{j}=\mathbf{1}}^{\mathbf{n}} \mathbf{B}_{\mathbf{j}}$. i.e., the summation of total supply must equal to the summation of total demand $[3]$.

\subsubsection{PROPOSED ALGORITHM:}

Step\#01:- Case (i) whenever $\sum_{\mathbf{i}} \mathbf{A}_{\mathbf{i}}>\sum_{\mathbf{j}} \mathbf{B}_{\mathbf{j}}$, add $\mathrm{C}_{\mathrm{ij}+1}=0 \forall$ dummy column based on the demand ( $\left.\mathrm{B}_{\mathbf{j}+1}\right)$, and calculate the absolute differences (penalty) between Initial \& Last cost cells of each column in transportation cost-matrix, (ignoring differences (penalty) of Initial \& Last cost cells of dummy column and all rows), then identify maximum penalty $\left(\mathrm{P}_{\mathrm{j}}\right)$ for allocation.

Case (ii) whenever $\sum_{\mathbf{i}} \mathbf{A}_{\mathbf{i}}<\sum_{\mathbf{j}} \mathbf{B}_{\mathbf{j}}$, add $\mathrm{C}_{\mathrm{ij}+1}=0 \forall$ dummy row based on the supply $\left(\mathrm{A}_{\mathrm{i}+1}\right)$, and calculate the absolute differences (penalty) between Initial \& Last cost cells of each row in transportation cost-matrix, (ignoring differences (penalty) of Initial \& Last cost cells of dummy row and all columns), then identify maximum penalty $\left(\mathrm{P}_{\mathrm{i}}\right)$ for allocation.

Mathematically,

$\mathbf{C}_{\mathrm{ij}+\mathbf{1}}=\mathbf{0} \forall$ dummy rows \& dummy columns.

$\mathbf{P}_{\mathbf{i}}=\left|\mathbf{C}_{\mathbf{1 n}}-\mathbf{C}_{\mathbf{m n}}\right| \forall$ rows penalty $\& \mathbf{P}_{\mathbf{j}}=\left|\mathbf{C}_{\mathbf{m} \mathbf{1}}-\mathbf{C}_{\mathbf{m n}}\right| \forall$ columns penalty.

Step\#02:- Choose the smallest cost cell $\mathrm{C}_{\mathrm{ij}}$ in row/column and allocate with selected maximum penalty $\left(\mathrm{P}_{\mathrm{i}} / \mathrm{P}_{\mathrm{j}}\right)$.

Step\#03:- "In case of ties", If two or more of the smallest cost cells $C_{i j}$ in row/column are same then select the top entry of them to allocate. If two or more of maximum penalty $\left(\mathrm{P}_{\mathrm{i}} / \mathrm{P}_{\mathrm{j}}\right)$ are same then select the smallest cost cell $\mathrm{C}_{\mathrm{ij}}$ in row/column.

Step\#04:- Repeat steps (1 to 3 ) for remaining transportation cost-matrix, continue this procedure until all the supply $\left(\mathrm{A}_{\mathrm{i}}\right)$ and demand $\left(\mathrm{B}_{\mathrm{j}}\right)$ are become zero.

Note: The dummy row/column should be allocated in last. 
Step\#05:- Finally, calculate minimizing the total cost of transportation.

i.e., $\mathbf{T} \operatorname{cost},\left\{\mathbf{Z}=\sum_{\mathbf{i}=1}^{\mathrm{m}} \sum_{\mathbf{j}=\mathbf{1}}^{\mathrm{n}} \mathbf{C}_{\mathbf{i j}} \mathbf{U}_{\mathrm{ij}}\right\}$.

\section{NUMERICAL EXAMPLES}

Consider the following different - sizes of both the cases of unbalanced transportation problems, selected from literature. We solve them using proposed algorithm and compare these results with the solution of NWCM, LCM \& MODI-Method.

3.1 Example\#1:- The Unbalanced-TP of case (i) Excess of accessibility $\sum_{\mathbf{i}} \mathbf{A}_{\mathbf{i}}>\sum_{\mathbf{j}} \mathbf{B}_{\mathbf{j}}$.

\begin{tabular}{|l|l|l|l|l|}
\hline Sources\Destinations & $\mathrm{D}_{1}$ & $\mathrm{D}_{2}$ & $\mathrm{D}_{3}$ & Supply $\left(\mathrm{A}_{\mathrm{i}}\right)$ \\
\hline $\mathrm{S}_{1}$ & 3 & 4 & 6 & 100 \\
\hline $\mathrm{S}_{2}$ & 7 & 3 & 8 & 80 \\
\hline $\mathrm{S}_{3}$ & 6 & 4 & 5 & 90 \\
\hline $\mathrm{S}_{4}$ & 7 & 5 & 2 & 120 \\
\hline Demand $\left(\mathrm{B}_{\mathrm{j}}\right)$ & 110 & 110 & 60 & $\sum 280 \backslash 390$ \\
\hline
\end{tabular}

Table: 3.1.1

\begin{tabular}{|l|l|l|l|l|l|}
\hline Sources Destinations & $\mathrm{D}_{1}$ & $\mathrm{D}_{2}$ & $\mathrm{D}_{3}$ & $\begin{array}{l}\text { Dummy } \\
\text { column }\end{array}$ & Supply $\left(\mathrm{A}_{\mathrm{i}}\right)$ \\
\hline $\mathrm{S}_{1}$ & 3 & 4 & 6 & 0 & 100 \\
\hline $\mathrm{S}_{2}$ & 7 & 3 & 8 & 0 & 80 \\
\hline $\mathrm{S}_{3}$ & 6 & 4 & 5 & 0 & 90 \\
\hline $\mathrm{S}_{4}$ & 7 & 5 & 2 & 0 & 120 \\
\hline Demand $\left(\mathrm{B}_{\mathrm{j}}\right)$ & 110 & 110 & 60 & 110 & $\sum 390 \backslash 390$ \\
\hline
\end{tabular}

Table: 3.1.2

\begin{tabular}{|l|l|l|l|l|l|}
\hline Sources\Destinations & $\mathrm{D}_{1}$ & $\mathrm{D}_{2}$ & $\mathrm{D}_{3}$ & $\begin{array}{l}\text { Dummy } \\
\text { column }\end{array}$ & Supply $\left(\mathrm{A}_{\mathrm{i}}\right)$ \\
\hline $\mathrm{S}_{1}$ & 3 & 4 & 6 & 0 & 100 \\
\hline $\mathrm{S}_{2}$ & 7 & 3 & 8 & 0 & 80 \\
\hline $\mathrm{S}_{3}$ & 6 & 4 & 5 & 0 & 90 \\
\hline $\mathrm{S}_{4}$ & 7 & 5 & $\mathbf{2}$ & 0 & $\begin{array}{l}120 \\
60\end{array}$ \\
\hline Demand $\left(\mathrm{B}_{\mathrm{j}}\right)$ & 110 & 110 & $\begin{array}{l}60 \\
0\end{array}$ & 110 & $\sum 330 \backslash 330$ \\
\hline Column penalty $\left(\mathrm{P}_{\mathrm{j}}\right)$ & $(4)$ & $(1)$ & $(4)$ & & \\
\hline
\end{tabular}

Table: 3.1 .3

\begin{tabular}{|c|c|c|c|c|}
\hline Sources\Destinations & $\mathrm{D}_{1}$ & $\mathrm{D}_{2}$ & $\begin{array}{l}\text { Dummy } \\
\text { column }\end{array}$ & Supply $\left(\mathrm{A}_{\mathrm{i}}\right)$ \\
\hline $\mathrm{S}_{1}$ & $\begin{array}{ll}3 & \\
& 100\end{array}$ & 4 & 0 & $\begin{array}{l}100 \\
0\end{array}$ \\
\hline $\mathrm{S}_{2}$ & 7 & 3 & 0 & 80 \\
\hline $\mathrm{S}_{3}$ & 6 & 4 & 0 & 90 \\
\hline $\mathrm{S}_{4}$ & 7 & 5 & 0 & 60 \\
\hline Demand $\left(B_{j}\right)$ & $\begin{array}{l}110 \\
10\end{array}$ & 110 & 110 & $\sum 230 \backslash 230$ \\
\hline Column penalty $\left(\mathrm{P}_{\mathrm{j}}\right)$ & (4) & (1) & & \\
\hline
\end{tabular}

Table: 3.1.4 


\begin{tabular}{|l|l|l|l|l|}
\hline Sources\Destinations & $\mathrm{D}_{1}$ & $\mathrm{D}_{2}$ & $\begin{array}{l}\text { Dummy } \\
\text { column }\end{array}$ & Supply $\left(\mathrm{A}_{\mathrm{i}}\right)$ \\
\hline $\mathrm{S}_{2}$ & 7 & $\mathbf{3}$ & 0 & $\begin{array}{l}80 \\
\mathbf{8 0}\end{array}$ \\
\hline $\mathrm{S}_{3}$ & 6 & 4 & 0 & 90 \\
\hline $\mathrm{S}_{4}$ & 7 & 5 & 0 & 60 \\
\hline Demand $\left(\mathrm{B}_{\mathrm{j}}\right)$ & 10 & $\begin{array}{l}110 \\
30\end{array}$ & 110 & $\sum 150 \backslash 150$ \\
\hline Column penalty $\left(\mathrm{P}_{\mathrm{j}}\right)$ & $(0)$ & $(2)$ & & \\
\hline
\end{tabular}

Table: 3.1 .5

\begin{tabular}{|c|c|c|c|c|}
\hline Sources\Destinations & $\mathrm{D}_{1}$ & $\mathrm{D}_{2}$ & $\begin{array}{c}\text { Dummy } \\
\text { column }\end{array}$ & Supply $\left(A_{i}\right)$ \\
\hline $\mathrm{S}_{3}$ & 6 & $\begin{array}{ll}4 & \\
& 30 \\
\end{array}$ & 0 & $\begin{array}{l}90 \\
60 \\
\end{array}$ \\
\hline $\mathrm{S}_{4}$ & 7 & 5 & 0 & 60 \\
\hline Demand $\left(B_{j}\right)$ & 10 & $\begin{array}{l}30 \\
0\end{array}$ & 110 & $\sum 120 \backslash 120$ \\
\hline Column penalty $\left(\mathrm{P}_{\mathrm{j}}\right)$ & (1) & $(1)$ & & \\
\hline
\end{tabular}

Table: 3.1.6

\begin{tabular}{|c|c|c|c|}
\hline Sources $\backslash$ Destinations & $\mathrm{D}_{1}$ & $\begin{array}{l}\text { Dummy } \\
\text { column }\end{array}$ & Supply $\left(A_{i}\right)$ \\
\hline $\mathrm{S}_{3}$ & $\begin{array}{ll}6 & \\
& 10 \\
\end{array}$ & 0 & $\begin{array}{l}60 \\
50\end{array}$ \\
\hline $\mathrm{S}_{4}$ & 7 & 0 & 60 \\
\hline Demand $\left(B_{j}\right)$ & $\begin{array}{l}10 \\
0 \\
\end{array}$ & 110 & $\sum 110 \backslash 110$ \\
\hline Column penalty $\left(\mathrm{P}_{\mathrm{j}}\right)$ & $(1)$ & & \\
\hline
\end{tabular}

Table: 3.1 .7

\begin{tabular}{|l|l|l|}
\hline Sources\Destinations & $\begin{array}{l}\text { Dummy } \\
\text { column }\end{array}$ & Supply $\left(\mathrm{A}_{\mathrm{i}}\right)$ \\
\hline $\mathrm{S}_{3}$ & $\mathbf{0} \quad \mathbf{5 0}$ & $\begin{array}{l}50 \\
0\end{array}$ \\
\hline $\mathrm{S}_{4}$ & $\mathbf{0} \quad \mathbf{6 0}$ & $\begin{array}{l}60 \\
0\end{array}$ \\
\hline Demand $\left(\mathrm{B}_{\mathrm{j}}\right)$ & $\begin{array}{l}110 \\
60\end{array}$ & $\sum 0 \backslash 0$ \\
& 0 & \\
\hline
\end{tabular}

Table: 3.1.8

$\mathrm{U}_{43}=60, \mathrm{U}_{11}=100, \mathrm{U}_{22}=80, \mathrm{U}_{32}=30, \mathrm{U}_{31}=10, \mathrm{U}_{34}=50, \& \mathrm{U}_{44}=60$.

Tcost, $Z=(2 * 60)+(3 * 100)+(3 * 80)+(4 * 30)+(6 * 10)+(0 * 50)+(0 * 60)=840 /-$

\section{Optimality Test}

Formulate an optimality test by MODI-Method, to find whether the obtained feasible solution is optimal or not. Here, the number of allocations is equal to $(m+n-1)=4+4-1=7$, hence optimality test can be achieved. Find $X$ 's and $Y$ 's values using the formula $X_{i}+Y_{j}=C_{i j} \forall$ allocated cells, and set $X_{1}=0$. Then, find $d_{i j}=\left(X_{i}+Y_{j}\right)$ $-\mathrm{C}_{\mathrm{ij}} \leq 0 \forall$ unallocated cells.

Hence all $\mathrm{d}_{\mathrm{ij}} \leq 0$, the solution given below is optimal. 


\begin{tabular}{|c|c|c|c|c|c|c|}
\hline Sources/Destinations & $\mathrm{D}_{1}$ & $\mathrm{D}_{2}$ & $\mathrm{D}_{3}$ & $\begin{array}{l}\text { Dummy } \\
\text { column }\end{array}$ & Supply $\left(A_{i}\right)$ & $\begin{array}{l}\text { Values } \\
\left(\mathrm{X}_{\mathrm{i}}\right)\end{array}$ \\
\hline $\mathrm{S}_{1}$ & $\begin{array}{ll}3 & \\
& 100\end{array}$ & $\begin{array}{lr}4 & 1 \\
& -3\end{array}$ & $\begin{array}{ll}6 & -1 \\
& -7\end{array}$ & $\begin{array}{ll}0 & -3 \\
& -3\end{array}$ & 100 & $X_{1}=0$ \\
\hline $\mathrm{S}_{2}$ & $\begin{array}{lr}7 & 5 \\
& -2\end{array}$ & $\begin{array}{ll}3 & \\
& 80\end{array}$ & $\begin{array}{lr}8 & 1 \\
& -7\end{array}$ & $\begin{array}{l}-1 \\
-1\end{array}$ & 80 & $\mathrm{X}_{2}=2$ \\
\hline $\mathrm{S}_{3}$ & 10 & 30 & $\begin{array}{ll}5 & 2 \\
& -3\end{array}$ & 50 & 90 & $\mathrm{X}_{3}=3$ \\
\hline $\mathrm{S}_{4}$ & $\begin{array}{cc}7 & 6 \\
& -1 \\
\end{array}$ & $\begin{array}{cc}5 & 4 \\
& -1 \\
\end{array}$ & $\begin{array}{ll}2 & \\
& 60 \\
\end{array}$ & 60 & 120 & $\mathrm{X}_{4}=3$ \\
\hline Demand $\left(\mathrm{B}_{\mathrm{j}}\right)$ & 110 & 110 & 60 & 110 & $\sum 3901390$ & \\
\hline Values $\left(\mathrm{Y}_{\mathrm{j}}\right)$ & $\mathrm{Y}_{1}=3$ & $Y_{2}=1$ & $Y_{3}=-1$ & $Y_{4}=-3$ & & \\
\hline
\end{tabular}

Table: 3.1.9

3.2 Example\#2:- The Unbalanced-TP of case (ii) Deficiency in accessibility $\sum_{\mathbf{i}} \mathbf{A}_{\mathbf{i}}<\sum_{\mathbf{j}} \mathbf{B}_{\mathbf{j}}$.

\begin{tabular}{|l|l|l|l|l|l|}
\hline Sources\Destinations & $\mathrm{D}_{1}$ & $\mathrm{D}_{2}$ & $\mathrm{D}_{3}$ & $\mathrm{D}_{4}$ & Supply $\left(\mathrm{A}_{\mathrm{i}}\right)$ \\
\hline $\mathrm{S}_{1}$ & 10 & 15 & 12 & 12 & 200 \\
\hline $\mathrm{S}_{2}$ & 8 & 10 & 11 & 9 & 150 \\
\hline $\mathrm{S}_{3}$ & 11 & 12 & 13 & 10 & 120 \\
\hline Demand $\left(\mathrm{B}_{\mathrm{j}}\right)$ & 140 & 120 & 80 & 220 & $\sum 560 \backslash 470$ \\
\hline
\end{tabular}

Table: 3.2.1

\begin{tabular}{|l|l|l|l|l|l|}
\hline Sources\Destinations & $\mathrm{D}_{1}$ & $\mathrm{D}_{2}$ & $\mathrm{D}_{3}$ & $\mathrm{D}_{4}$ & Supply $\left(\mathrm{A}_{\mathrm{i}}\right)$ \\
\hline $\mathrm{S}_{1}$ & 10 & 15 & 12 & 12 & 200 \\
\hline $\mathrm{S}_{2}$ & 8 & 10 & 11 & 9 & 150 \\
\hline $\mathrm{S}_{3}$ & 11 & 12 & 13 & 10 & 120 \\
\hline Dummy row & 0 & 0 & 0 & 0 & 90 \\
\hline Demand $\left(\mathrm{B}_{\mathrm{j}}\right)$ & 140 & 120 & 80 & 220 & $\sum 560 \backslash 560$ \\
\hline
\end{tabular}

Table: 3.2.2

\begin{tabular}{|l|l|l|l|l|l|l|}
\hline Sources|Destinations & $\mathrm{D}_{1}$ & $\mathrm{D}_{2}$ & $\mathrm{D}_{3}$ & $\mathrm{D}_{4}$ & Supply $\left(\mathrm{A}_{\mathrm{i}}\right)$ & $\begin{array}{l}\text { Row penalty } \\
\left(\mathrm{P}_{\mathrm{i}}\right)\end{array}$ \\
\hline $\mathrm{S}_{1}$ & $\mathbf{1 0}$ & 15 & 12 & 12 & $\begin{array}{l}200 \\
60\end{array}$ & $(2)$ \\
\hline $\mathrm{S}_{2} \mathbf{1 4 0}$ & 8 & 10 & 11 & 9 & 150 & $(1)$ \\
\hline $\mathrm{S}_{3}$ & 11 & 12 & 13 & 10 & 120 & $(1)$ \\
\hline Dummy row & 0 & 0 & 0 & 0 & 90 & \\
\hline Demand $\left(\mathrm{B}_{\mathrm{j}}\right)$ & 140 & 120 & 80 & 220 & $\sum 420 \backslash 420$ & \\
\hline
\end{tabular}

Table: 3.2.3

\begin{tabular}{|c|c|c|c|c|c|}
\hline Sources\Destinations & $\mathrm{D}_{2}$ & $\mathrm{D}_{3}$ & $\mathrm{D}_{4}$ & Supply $\left(\mathrm{A}_{\mathrm{i}}\right)$ & $\begin{array}{l}\text { Row penalty } \\
\left(\mathrm{P}_{\mathrm{i}}\right)\end{array}$ \\
\hline $\mathrm{S}_{1}$ & 15 & $\begin{array}{ll}12 & \\
& 60 \\
\end{array}$ & 12 & $\begin{array}{l}60 \\
0\end{array}$ & (3) \\
\hline $\mathrm{S}_{2}$ & 10 & 11 & 9 & 150 & (1) \\
\hline $\mathrm{S}_{3}$ & 12 & 13 & 10 & 120 & (2) \\
\hline Dummy row & 0 & 0 & 0 & 90 & \\
\hline Demand $\left(B_{j}\right)$ & 120 & $\begin{array}{l}80 \\
20 \\
\end{array}$ & 220 & $\sum 360 \backslash 360$ & \\
\hline
\end{tabular}

Table: 3.2.4 


\begin{tabular}{|c|c|c|c|c|c|}
\hline Sources\Destinations & $\mathrm{D}_{2}$ & $\mathrm{D}_{3}$ & $\mathrm{D}_{4}$ & Supply $\left(\mathrm{A}_{\mathrm{i}}\right)$ & $\begin{array}{l}\text { Row penalty } \\
\left(\mathrm{P}_{\mathrm{i}}\right)\end{array}$ \\
\hline $\mathrm{S}_{2}$ & 10 & 11 & 9 & 150 & (1) \\
\hline $\mathrm{S}_{3}$ & 12 & 13 & $\begin{array}{ll}10 & \\
& 120 \\
\end{array}$ & $\begin{array}{l}120 \\
0\end{array}$ & (2) \\
\hline Dummy row & 0 & 0 & 0 & 90 & \\
\hline Demand $\left(\mathrm{B}_{\mathrm{j}}\right)$ & 120 & 20 & $\begin{array}{l}220 \\
100\end{array}$ & $\sum 240 \backslash 240$ & \\
\hline
\end{tabular}

Table: 3.2.5

\begin{tabular}{|l|l|l|l|l|l|}
\hline Sources\Destinations & $\mathrm{D}_{2}$ & $\mathrm{D}_{3}$ & $\mathrm{D}_{4}$ & Supply $\left(\mathrm{A}_{\mathrm{i}}\right)$ & $\begin{array}{l}\text { Row penalty } \\
\left(\mathrm{P}_{\mathrm{i}}\right)\end{array}$ \\
\hline $\mathrm{S}_{2}$ & 10 & 11 & $\mathbf{9}$ & $\begin{array}{l}150 \\
50\end{array}$ & $(1)$ \\
\hline Dummy row & 0 & 0 & 0 & 90 & \\
\hline Demand $\left(\mathrm{B}_{\mathrm{j}}\right)$ & 120 & 20 & $\begin{array}{l}100 \\
0\end{array}$ & $\sum 140 \backslash 140$ & \\
\hline
\end{tabular}

Table: 3.2.6

\begin{tabular}{|l|l|l|l|l|}
\hline Sources $\backslash$ Destinations & $\mathrm{D}_{2}$ & $\mathrm{D}_{3}$ & Supply $\left(\mathrm{A}_{\mathrm{i}}\right)$ & $\begin{array}{l}\text { Row penalty } \\
\left(\mathrm{P}_{\mathrm{i}}\right)\end{array}$ \\
\hline $\mathrm{S}_{2}$ & $\mathbf{1 0}$ & 11 & $\begin{array}{l}50 \\
0\end{array}$ & $(1)$ \\
\hline Dummy row & 0 & 0 & 90 & \\
\hline Demand $\left(\mathrm{B}_{\mathrm{j}}\right)$ & $\begin{array}{l}\mathbf{5 0} \\
70\end{array}$ & 20 & $\sum 90190$ & \\
\hline
\end{tabular}

Table: 3.2.7

\begin{tabular}{|c|c|c|c|}
\hline SourceslDestinations & $\mathrm{D}_{2}$ & $\mathrm{D}_{3}$ & Supply $\left(A_{i}\right)$ \\
\hline Dummy row & $\begin{array}{ll}0 & \\
& 70\end{array}$ & \begin{tabular}{|ll}
${ }^{\circ}$ & \\
&
\end{tabular} & $\begin{array}{l}90 \\
0\end{array}$ \\
\hline Demand $\left(B_{j}\right)$ & $\begin{array}{l}70 \\
0\end{array}$ & $\begin{array}{l}20 \\
0\end{array}$ & $\sum 0 \backslash 0$ \\
\hline
\end{tabular}

Table: 3.2.8

$$
\mathrm{U}_{11}=140, \mathrm{U}_{13}=60, \mathrm{U}_{34}=120, \mathrm{U}_{24}=100, \mathrm{U}_{22}=50, \mathrm{U}_{42}=70, \& \mathrm{U}_{43}=20 .
$$

Tcost, $Z=(10 * 140)+(12 * 60)+(10 * 120)+(9 * 100)+(10 * 50)+(0 * 70)+(0 * 20)=4,720 /-$

Optimality test, the number of allocations is equal to $(\mathrm{m}+\mathrm{n}-1)=4+4-1=7$. Hence all $\mathrm{d}_{\mathrm{ij}} \leq 0$ the solution given below is optimal.

\begin{tabular}{|c|c|c|c|c|c|c|}
\hline SourceslDestinations & $\mathrm{D}_{1}$ & $\mathrm{D}_{2}$ & $\mathrm{D}_{3}$ & $\mathrm{D}_{4}$ & Supply $\left(\mathrm{A}_{\mathrm{i}}\right)$ & $\begin{array}{l}\text { Values } \\
\left(\mathrm{X}_{\mathrm{X}}\right)\end{array}$ \\
\hline $\mathrm{S}_{1}$ & 10 140 & $\begin{array}{cc}15 & 12 \\
& -3 \\
\end{array}$ & $\begin{array}{ll}12 & \\
& 60 \\
\end{array}$ & $\begin{array}{cc}12 & 11 \\
& -1 \\
\end{array}$ & 200 & $\mathrm{X}_{1}=0$ \\
\hline$\overline{S_{2}}$ & $\begin{array}{l}8 \\
0 \\
\end{array}$ & $\begin{array}{ll}10 & \\
& 50 \\
\end{array}$ & $\begin{array}{cc}11 & 10 \\
& -1 \\
\end{array}$ & $\begin{array}{ll}9 & \\
& 100 \\
\end{array}$ & 150 & $X_{2}=-2$ \\
\hline $\mathrm{S}_{3}$ & $11{ }^{11}{ }^{9}$ & $\begin{array}{cc}12 & 11 \\
& -1\end{array}$ & $\begin{array}{cc}13 & 11 \\
& -2\end{array}$ & 10 120 & 120 & $X_{3}=-1$ \\
\hline Dummy row & $\begin{array}{ll}0 & -2 \\
& -2 \\
\end{array}$ & 70 & 20 & $\begin{array}{ll}0 & -1 \\
& -1\end{array}$ & 90 & $X_{4}=-12$ \\
\hline Demand $\left(\mathrm{B}_{\mathrm{j}}\right)$ & 140 & 120 & 80 & 220 & $\sum 560 \backslash 560$ & \\
\hline Values $\left(\mathrm{Y}_{\mathrm{j}}\right)$ & $Y_{1}=10$ & $Y_{2}=12$ & $Y_{3}=12$ & $\mathrm{Y}_{4}=11$ & & \\
\hline
\end{tabular}

Table: 3.2.9

\section{COMPARISON OF RESULTS TABLE \& DISCUSSION:}

Consider the Table 4.1.1, we have solved the different real life problems by the proposed method. The following examples of case (i) 1,3,5 and case (ii) 2, 4, 6 are solved and several others, selected from literature. Also tested the performance of proposed method in comparison to NWC-Method, LC-Method \& MODI-Method, and it can 
be clearly seen that the proposed method is same as the optimal results.

\begin{tabular}{|c|c|c|c|c|c|c|}
\hline $\begin{array}{l}\text { Numerical } \\
\text { Examples }\end{array}$ & Rows & Columns & $\begin{array}{l}\text { NWC- } \\
\text { Method }\end{array}$ & $\begin{array}{l}\text { LC- } \\
\text { Method }\end{array}$ & $\begin{array}{l}\text { Proposed- } \\
\text { Method }\end{array}$ & $\begin{array}{l}\text { Optimal } \\
\text { MODI Method }\end{array}$ \\
\hline Ex:1 [Ref: 8] & 4 & 4 & 1,010 & 990 & $\mathbf{8 4 0}$ & $\mathbf{8 4 0}$ \\
\hline Ex:2 [Ref: 3] & 4 & 4 & 5,070 & 5,260 & $\mathbf{4 , 7 2 0}$ & $\mathbf{4 , 7 2 0}$ \\
\hline Ex:3 [Ref: 6] & 4 & 6 & 19,700 & 12,100 & $\mathbf{1 1 , 5 0 0}$ & $\mathbf{1 1 , 5 0 0}$ \\
\hline Ex:4 [Ref: 9] & 4 & 4 & 3,550 & 3,225 & $\mathbf{3 , 1 0 0}$ & $\mathbf{3 , 1 0 0}$ \\
\hline Ex:5 [Ref: 1] & 2 & 4 & 31,875 & 20,875 & $\mathbf{1 7 , 8 7 5}$ & $\mathbf{1 7 , 8 7 5}$ \\
\hline Ex:6 [Ref: 4] & 5 & 3 & 14,140 & 12,550 & $\mathbf{1 1 , 7 2 0}$ & $\mathbf{1 1 , 7 2 0}$ \\
\hline
\end{tabular}

\section{Table: 4.1.1}

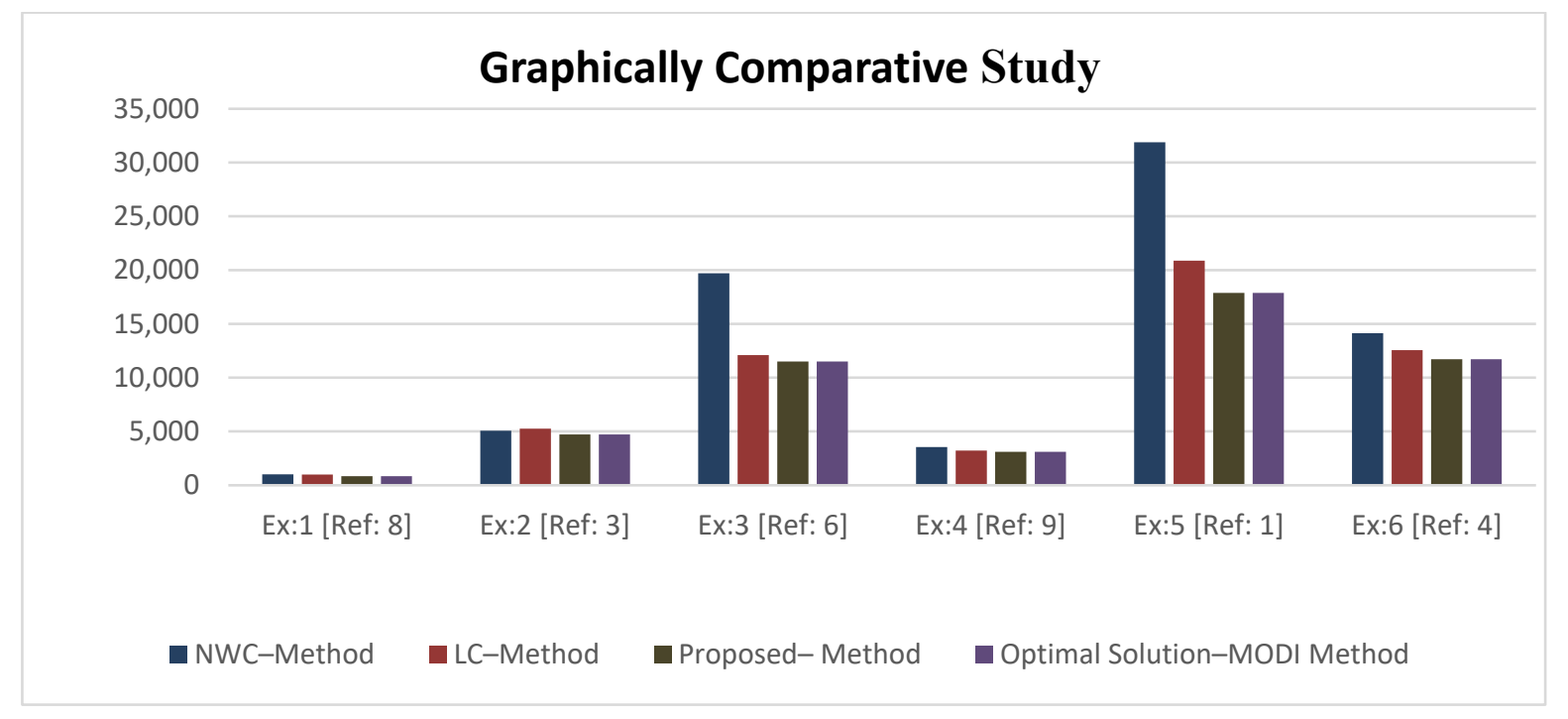

Fig: 1 Results are summarized in Table 4.1.1.

\section{CONCLUSION}

In this paper, we have promoted an improved algorithm for obtaining better optimal solution of unbalanced transportation problems. According to work computation, the proposed method is easy and less arithmetical calculation required to get optimal solution compared to existing MODI-Method. Also a comparison of proposed algorithm is made with NWC-Method, LC-Method and MODI-Method. Various numerical examples of different - sizes of both the cases are tested and critically observed that the proposed algorithm provide optimal solution directly.

\section{REFERENCES}

[1] S. Aramuthakannan \& P. R. Kandasamy (2016). “Application of Revised Distribution Method for Finding Optimal Solution of Unbalanced Transportation Problems”. PARIPEX- Indian Journal of Research, Vol.5, No.1, pp.32-34.

[2] Priyanka Malviya \& Dr. Sushma Jain (2018). "Modified form of Average Transportation Cost Method (ATCM) - an Efficient Method for Find an Initial Basic Feasible Solution for Transportation Problem". International Journal of Mathematics Trends and Technology (IJMTT), Vol.59, No.1, pp.1-3.

[3] T. Geetha \& N. Anandhi (2018). "Method for Solving Unbalanced Transportation Problems using Standard Deviations". International Journal of Pure and Applied Mathematics, Vol.119, No.16, pp.4971-4989.

[4] A. K. M. Selim Reza, A. R. M. Jalal Uddin Jamali \& Bristy Biswas (2019). "A Modified Algorithm for Solving Unbalanced Transportation Problems". Journal of Engineering Science (JES) an International Journal, Vol.10, No.1, pp.93-101.

[5] A. P. Verma, S. K. Kataria \& Sons (2005). “Operations Research”. Third Edition, pp.1-1200.

[6] Mollah Mesbahuddin Ahmed, Abu Sadat Muhammad Tanvir, Shirin Sultana, Sultan Mahmud \& Md. Sharif Uddin (2014). "An Effective Modification to Solve Transportation Problems: A Cost Minimization Approach”. Annals of Pure and Applied Mathematics, Vol.6, No.2, pp.199-206.

[7] I. A. Rajper, S. F. Shah \& A. S. Qureshi (2019). "Development of a New Algorithm for Optimal Solution of Transportation Problems". Mathematical Theory and Modeling ISSN: 2224-5804 (Paper), Vol.9, No.1, pp.86-93. 
[8] Z.A.M.S. Juman \& N.G.S.A. Nawarathne (2019). "An Efficient Alternative Approach to Solve a Transportation Problem". Ceylon Journal of Science, Vol.48, No.1, pp.19-29.

[9] Abdur Rashid (2017). "An Alternative Approach for Solving Unbalanced Assignment Problems". Jahangirnagar University Journal of Science-JUJS, Vol.40, No.2, pp.45-56.

[10] M. S. R. Shaikh, S. F. Shah \& Z. Memon (2018). “An Improved Algorithm to Solve Transportation Problems for Optimal Solution". Mathematical Theory and Modeling ISSN: 2224-5804 (Paper), Vol.8, No.8, pp.1-8.

[11] A. Sridhar \& R. Allah Pitchai (2018). "New Approach to Solve Unbalanced Transportation Problems using Vogel's Approximation Method”. International Journal of Mathematical Archive, Vol.9, No.11, pp.20-24.

[12] Nigus Girmay \& Tripti Sharma (2013). "Balance an Unbalanced Transportation Problem by a Heuristic Approach". International Journal of Mathematics and Its Applications (IJMAA), Vol.1, No.1, pp.13-19.

[13] Abdul Quddoos, Shakeel Javaid \& M. M. Khalid (2012). "A New Method for Finding an Optimal Solution for Transportation Problems". International Journal on Computer Science and Engineering (IJCSE), Vol.4, No.7, pp.1271-1273.

[14] Rajendra B. Patel (2018). "Solution of Transportation Problem with ASM Method and Comparison with Existing Methods for Optimal Solution". International Journal of Advance Engineering and Research Development (IJAERD), Vol.5, No.2, pp.84-86.

[15] Palanivel M \& Suganya M (2018). "A New Method To Solve Transportation Problem - Harmonic Mean Approach”. Engineering Technology Open Access Journal (ETOAJ), Vol.2, No.3, pp.1-3.

[16] Ravi Kumar R, Radha Gupta \& O. Karthiyayini (2018). "A New Approach to Find the Initial Basic Feasible Solution of a Transportation Problem". International Journal of Research - GRANTHAALAYAH, Vol.6, No.5, pp.321-325.

[17] A. Seethalakshmy \& N. Srinivasan (2017). "A New Approach to obtain an Optimal Solution for the Unbalanced Assignment Problem”. International Journal of Pure and Applied Mathematics, Vol.114, No.6, pp.77-85.

[18] M. A. Metlo, M. A. Solangi \& S. A. Memon (2016). "Modified North-West Corner Method for the Optimal Solution for Transportation Problems”. Sindh University Research Journal-SURJ (Science Series), Vol.48, No.4, pp.793-796.

[19] Qazi Shoeb Ahmed (2020). “A New Approach for Finding the Initial Solution of the Unbalanced Transportation Problem”. Asian Journal of Business and Management (ISSN: 2321-2802) Vol.8, No.4, pp.49-51.

[20] R. Murugesan \& T. Esakkiammal (2020). "TERM - A Very Simple and Efficient Method to Solve Assignment Problems". Applied Mathematical Sciences, Vol.14, No.17, pp.801-809. 